\title{
The Method of Information Influence on a Subject when Forming an Ensemble of Competencies in a Preset Time
}

\author{
Inessa A. Vorobeva* \\ Lipetsk State Pedagogical University \\ Named After P.P. Semenov-Tyan-Shan \\ 42 Lenin Str., Lipetsk, 398020, Russia
}

Received 21.05.2016, received in revised form 30.11.2017, accepted 13.01.2018

The article deals with the process of information interaction between a subject and an adaptive multiagent information system, presented as a set of operations for building networks, at the level of scientists at the entry level, assessing the current levels of their skills and operational reorganization of the network in accordance with the assessment of the level of their preparedness.

Keywords: the set of paths of dialogue script, adaptive multiagent system, an adaptive dialogue, competence.

Citation: Vorobeva I.A. The method of information influence on a subject when forming an ensemble of competencies in a preset time, J. Sib. Fed. Univ. Eng. technol., 2018, 11(1), 48-51. DOI: 10.17516/1999-494X-0007.

\section{Метод организации информационного воздействия на субъект при формировании множества компетенций за заданное время}

\author{
И.А. Воробьева \\ Липецкий государственный педагогический университет \\ имени П.П. Семенова-Тян-Шанского \\ Россия, 398020, Липецьк, ул. Ленина, 42
}

В статье рассмотрен процесс информационного взаимодействия субъекта с адаптивной мультиагентной информационной системой, представленный в виде совокупности операций по построению сети диалоговых процедур на основе учёта начального уровня квалификации субъекта, оценки текущчего уровня его квалификации и оперативной перестройки сети в соответствии с оценкой его уровня подготовленности.

(C) Siberian Federal University. All rights reserved

* Corresponding author E-mail address: vobi@bk.ru 
Ключевые слова: множество траекторий сиенария диалога, адаптивная мультиагентная система, адаптивный диалог, компетенции.

В последнее время подготовка специалиста стала одной из ключевых тем не только при исследовании педагогических подходов к этому процессу, но и при создании автоматизированных систем, учитывающих индивидуальные возможности субъекта для оптимизации времени и материальных ресурсов для его подготовки. В настоящей статье рассмотрен способ воздействия на субъект на основе выбора оптимального состава сценария в ходе организации диалога «субъект - ЭВМ».

Допустим, что из точки инициализации $\mathrm{x}_{0}$ в данную точку можно попасть единственным путем (траекторией), причем он состоит из $\mathrm{k}(\mathrm{k}>0)$ дуг, что соответствует $\mathrm{k}$ шагам диалога «человек-ЭВМ». Будем говорить тогда, что данный кадр сценария k-й кратности. Длину наибольшей траектории $\mathrm{d}_{\mathrm{x}}$, соединяющей $\mathrm{x}_{0} \mathrm{c} \mathrm{x}_{\mathrm{k}}$ по всем $\mathrm{k}$, назовем глубиной $\mathrm{K}$ сценария директивного ГДП G(X,F).

Будем говорить, что сценарий В вложен в сценарий $\mathrm{A}, \mathrm{B} \subset \mathrm{A}$, если множество траекторий сценария В мономорфно множеству траекторий сценария А, при условии, что элементы инициализации у обоих сценариев совпадают. Сценарий $\mathrm{S}$ назовем равномощным, если число траекторий, выходящих из элементов одной и той же кратности, одинаково. Сценарий, содержащий только точку инициализации диалога, будем также считать равномощным [1].

Каждому равномощному сценарию $\mathrm{S}$ глубины $\mathrm{k}>1$ припишем набор $\left(v_{1}, v_{2}, \ldots, v_{\mathrm{k}}\right)$, где $v_{\mathrm{i}}$ - соответствует числу траекторий, исходящих из произвольного элемента $(\mathrm{i}-1), \mathrm{i}=\overline{1, \mathrm{k}} ; \mathrm{k}-$ кратности. Причем $\mathrm{S}$ - равномощный сценарий с набором $\left(v_{1}, v_{2}, \ldots, v_{\mathrm{k}}\right)$ будет вложен в равномощный сценарий с набором $\left(\mu_{1}, \mu_{2}, \ldots, \mu_{\mathrm{n}}\right)$ тогда и только тогда, когда $\mathrm{k}<\mathrm{r}$ и $v_{\mathrm{i}}<\mu_{\mathrm{j}}, \mathrm{i}=\overline{1, \mathrm{k}}, \mathrm{j}=\overline{1, \mathrm{r}}$. Тогда два равномощных сценария, имеющих одинаковые номера, изоморфны.

Суммируя по всему и учитывая одноэлементный равномощный сценарий, получим следующую рекуррентную формулу:

$$
\sigma(1)=1, \sigma(\mathrm{n})=1+\sigma(\mathrm{n}-1)+\sigma\left(\left[\frac{\mathrm{n}-1}{2}\right]\right)+\mathrm{K}+\sigma\left(\left[\frac{\mathrm{n}-1}{\mathrm{n}}\right]\right) .
$$

Формула (1) - для расчета числа $\sigma(\mathrm{n})$ равномощных сценариев с конечным числом шагов диалога $\leq$ n. Очевидно, что количество равномощных сценариев с числом шагов диалога $\leq \mathrm{n}$ и таких, что из точки инициализации диалога выходит в точности $v, v=\overline{1, \mathrm{n}-1}$ дуг, равно $\sigma([\mathrm{n}-1] / v)$.

Зададимся равномощными сценариями $\mathrm{S}$ и $\mathrm{T}$, у которых одинаковая глубина $\mathrm{K}$, с соответствующими наборами $\left(v_{1}, v_{2}, \ldots, v_{k}\right)$ и $\left(\mu_{1}, \mu_{2}, \ldots, \mu_{n}\right)$. Назовем сценарий $\mathrm{S}$ короче сценария $\mathrm{T}, \mathrm{S}<\mathrm{T}$, если номер $\left(v_{1}, v_{2}, \ldots, v_{\mathrm{k}}\right)$ меньше номера $\left(\mu_{1}, \mu_{2}, \ldots, \mu_{\mathrm{n}}\right)$ справа в лексикографическом смысле, т.е. $\mathrm{i}=\max$, для которого $v_{\mathrm{i}} \neq \mu_{\mathrm{j}}$, такого, что $v_{\mathrm{i}}<\mu_{\mathrm{j}}$.

Зададимся теперь произвольным сценарием $\mathrm{S}$ и определим А с произвольным числом шагов диалога. Вычислим число попарно неизоморфных вложенных в А равномерных сценариев.

Алгоритм построения $\mathrm{N}$-полного сценария

1. Построить $\mathrm{N}$-полный сценарий.

2. Показать, что он является минимальным. 
3. Исследовать возможность построения универсального сценария для произвольного конечного множества равномощных сценариев.

Пусть $\mathrm{N}$ - множество всех директивных сценариев с одной вершиной инициализации и счетным множеством шагов диалога $\mathrm{n}, \mathrm{n}=\overline{1, \mathrm{n}}$. Будем использовать индуктивное построение $\mathrm{N}$-полного сценария. Пусть $\mathrm{W}_{1}$ - сценарий, состоящий только из элемента инициализации. Пусть для каждого $\mathrm{k}<\mathrm{n}$ уже есть сценарий $\mathrm{W}_{\mathrm{k}}$. Сценарий строим следующим образом.

Эman I. Из точки инициализации диалога $\mathrm{x}_{0}$ проведем $\mathrm{n}-1$ траекторий к элементам $\left(\mathrm{x}_{1}, \mathrm{x}_{2}, \ldots, \mathrm{x}_{\mathrm{n}-1}\right)$.

Этап II. Положим сценарий $\mathrm{W}_{\mathrm{n}}\left(\mathrm{x}_{\mathrm{i}}\right)$ равным сценарию $\mathrm{W}[(\mathrm{n}-1) / \mathrm{i}]$ для каждого $\mathrm{i}, 1 \leq \mathrm{i} \leq \mathrm{n}-1$. Тогда число элементов $\sigma(\mathrm{n})$ минимального универсального $\mathrm{N}$-полного сценария равно числу равномощных сценариев с числом шагов диалога $\leq \mathrm{n}$.

Обозначим через $\sigma(\mathrm{n})$ конечное множество равномощных сценариев. Найдем минимальный универсальный сценарий для $\sigma(\mathrm{n})$.

Рассмотрим множество $\mathrm{R}$, состоящее из элемента инициализации $\mathrm{x}_{0}$, и множества наборов $\left(v_{1}, v_{2}, \ldots, v_{k}\right)$, характеризующих те равномощные сценарии, которые могут быть вложены в какой-либо сценарий из $\sigma(\mathrm{n})$. Проведем далее траектории из $\mathrm{x}_{0}$ в $\left(v_{1}, v_{2}, \ldots, v_{\mathrm{k}}\right)$, полагая, что набор $\left(v_{1}, v_{2}, \ldots, v_{\mathrm{k}+1}\right)$ продолжает набор $\left(v_{1}, v_{2}, \ldots, v_{\mathrm{k}}\right)$. Обозначим сценарий такого типа через $\mathrm{A}\left(\sigma_{\mathrm{n}}\right)$.

Сценарий $\mathrm{A}\left(\sigma_{\mathrm{n}}\right)$ является искомым универсальным сценарием для множества $\sigma(\mathrm{n})$. При этом для $\sigma_{\mathrm{n}} \subset \sigma_{\mathrm{N}}$ имеем включение $\mathrm{A}\left(\sigma_{\mathrm{n}}\right) \subset \mathrm{A}\left(\sigma_{\mathrm{N}}\right)$, а для одноэлементных сценариев $\sigma_{1}=\{\mathrm{R}\}$, $\mathrm{A}\left(\sigma_{1}\right)$ изоморфно $\mathrm{R}$.

Для оценки эффективности сценария будем использовать алгоритм построения остовного дерева в целях определения наилучшего варианта. В теории для решения подобной задачи используется следующий алгоритмы: алгоритм Краскала и Гомори-Ху. Будем использовать алгоритм Краскала, так как он предлагает более простой и эффективный алгоритм для его программой реализации.

Итак, алгоритм Краскала [2]:

1. Осуществляется сортировка ребер графа по возрастанию весов.

2. Полагается, что каждой компоненте связности соответствует своя вершина.

3. Отсортированные ребра рассматриваются в порядке возрастания весов, для чего выполняются следующие действия:

- Ребро, у которого вершины лежат в разных компонентах связности, добавляется к минимальному остовному дереву, при этом компоненты связности объединяются в одну.

- Ребро, у которого вершины лежат в одной компоненте связности, исключается из рассмотрения.

4. При наличии нерассмотренных ребер и необъединенных компонентов связности переходят к третьему шагу. В противном случае алгоритм заканчивается.

Рассмотрим заданный ранее матрицей весов $W$ граф. Можно видеть, что работать непосредственно с матрицей неудобно, что обнаруживаем уже на этапе упорядочивания ребер по весу. Поэтому целесообразно вначале выделить массив ребер с соответствующими весами.

В первую очередь будем рассматривать простые массивы. Каждое ребро должно иметь начальную вершину, конечную вершину и вес.

$$
-50-
$$


Для задания набора ребер используется два массива E: array [1..m,1..2] of integer, здесь $m$ - количество ребер $\left(m<n^{2}-n+1\right.$, где $n$ - количество вершин), и массив $E W$ : array [1..m] of $r e a l$, тогда ребро $e_{i}$, соединяющее вершины $u, v$ с весом $w_{i}$ - будет соответствовать элементам $E[i, 1]=u, E[i, 2]=v, E W[i]=w$. Таким образом, до начала непосредственно поиска минимального остовного дерева необходимо пройти матрицу весов $W$ и заполнить массивы $E$ и $E W$.

Далее преобразованием весовой матрицы набор ребер упорядочивается по неубыванию весов. Если граф изначально задан с помощью списка ребер, то предыдущую часть алгоритма можно опустить. Для упорядочивания используется алгоритм сортировки «всплывающие пузырьки», который при необходимости позволяет использовать другие способы упорядочивания.

Таким образом, полученные результаты в виде упорядоченного набора вершин, отсортированных по заданному критерию, представляют собой оптимальный набор информационных элементов, определяющих индивидуальный учебный курс подготовки.

\section{Список литературы}

[1] Кузнецов И.П. Расширенные семантические сети для представления и обработки знаний. Системы и средства информатики, М.: РАН, 1993, 4, 70-84. [Kuznecov I.P. Advanced semantic networks for the representation and processing of knowledge. Systems and means of informatics, M., RAN, 1993, 4, 70-84 (in Russian)].

[2] Хартли Д. К вопросу об оценке обучающих программ. Кибернетика и проблемы обучения. Ред. и пред. А.И. Берга, М., Прогресс, 1970, 350-387. [Hartli D. On the issue of evaluation of training programs. Cybernetics and learning problems: a collection. Revision and provision A.I. Berga, M.: Progress, 1970, 350-387 (in Russian)]. 